\title{
Design of a New Non-Singular Robust Control Using Synergetic Theory for DC-DC Buck Converter
}

\author{
Yakoub Nettari, Serkan Kurt \\ Department of Electronics and Communications Engineering, Yıldız Technical University Faculty of Electrical and Electronics Engineering, İstanbul, Turkey
}

Cite this article as: Y. Nettari, S. Kurt, "Design of a New Non-Singular Robust Control Using Synergetic Theory for DC-DC Buck Converter", Electrica, vol. 18, no: 2, pp. 292-299, 2018.

\begin{abstract}
DC-DC converters control has gained much attention because of their broad uses in various fields, ranging from hand-held calculators to sophisticated airborne vehicles. Robustness in control systems, in spite of parametric variations, is an absolute requirement in many such applications. We propose a new controller to realize a robust performance despite the uncertainties on system parameter values. The controller employs an adaptive non-singular finite-time synergetic control method to tackle disturbances, which enhances the robustness and enables better performance during the transient phase compared to the terminal sliding mode control. A finite-time convergence is therefore achieved, while Lyapunov synthesis guarantees stability. Extensive simulation results of the DC-DC converter under harsh operating conditions confirm the effectiveness of the proposed controller.
\end{abstract}

Keywords: Synergetic control, finite time convergence, adaptive, DC-DC converter, sliding mode

\section{Corresponding Author: \\ Yakoub Nettari}

\section{E-mail:}

yayanet23@gmail.com,

skurt@yildiz.edu.tr

Received: 07.03 .2018

Accepted: 22.06 .2018

(c) Copyright 2018 by Electrica

Available online at

http://electrica.istanbul.edu.tr

DOI: 10.26650/electrica.2018.03052

\section{Introduction}

There is an ever-increasing demand for sound robust controller converters due to their wide variety of applications. Despite the undesirable chattering phenomenon, one of the most widely used techniques designed for a robust controller is the sliding mode control, used in a collection of applications varying from dishwashers to military equipment. Numerous efforts have been made to reduce or eliminate this major drawback of chattering. Listed among some of the most critical methods are Slotine's bounding layer and Levant's higher-order sliding modes [1, 2].

Sliding mode, as a proven robust control approach, led to a significant effort to deal with its main drawback, chattering, due to its discontinuous law constituent, many techniques have been proposed in an attempt to eliminate the chattering phenomenon but these have mostly been harmful to the robustness of the system [1, 3, 4]. Synergetic control, similar to SMC, is based on the concept of forcing a system to the selected manifold by chosen dynamics. Furthermore, synergetic theory achieves identical performance as SMC without carrying the deficiency of chattering to accomplish a non-chattering desired performance, and synergetic control elaborates a manifold after choosing a pertinent macro variable.

It was shown that we can choose the macro-variable function in Synergetic Control as a function of system state variables precisely as sliding surface in Sliding Mode Control [5]. Even though there are similarities between those techniques in terms of decoupling and system order reduction, its chatter-free characteristics enable chatter-free operation, which increases its practical value. Nevertheless, convergence attained via this approach is only asymptotic. We propose to build on recent work on finite time convergence and apply a similar scheme to elaborate a terminal synergetic controller for a DC-DC buck converter [6].

The latter not only has the advantage of fast convergence but also offers a small steady state error $[7,8]$. However, some terms in conventional finite time sliding mode or synergetic control have the drawback of singularity [9]. Nonsingular terminal synergetic control can prevent singu- 
larity, but the disturbances variety must be limited, to define the switching gain value [10-11].

Another important approach in dealing with uncertain nonlinear systems is adaptive control, which has proven very useful in tackling control problems $[12,13]$. This study proposes an adaptive estimation scheme, which is integrated with the controller that realizes fast convergence even in the presence of boundary disturbances. The proposed controller also overcomes the singularity problems which are a feature of synchronous terminal synergetic control design.

This paper is organized as follows: in Sections II and III, we discuss general synergetic controller procedure and give a quick overview of DC-DC buck converter modeling respectively. Then, in Sections IV and V, we cover the topic of terminal synergetic control design, which is followed by a detailed discussion of adaptive terminal synergetic control, and a development of adaptive laws. Next, in Section VI we present simulation results, and finally in Section VII we discuss our results/findings and make our concluding remarks.

\section{Synergetic Control}

Though introduced relatively recently, synergetic control, has a lot of advantages compared to Sliding mode control, and it has achieved full acceptance both from the control community as well as from industry, as demonstrated by its extensive use in power electronics and industrial battery charging [14-18].

We briefly recall that synergetic control synthesis consists of the following steps:

- We define a macro variable as:

$\psi(t)=\psi(t, \chi)$

which forces the system to operate on a pre-selected manifold, $\psi=0$. The designer selects the characteristics of this macro-variable according to the control specifications.

- The dynamic evolutions of the macro-variables are fixed to ensure a designer chosen constraint, such as

$T \psi+\psi=0$

where $T>0$ is, the design parameter, which defines the speed of convergence to $\psi=0$.

- The control law is synthesized according to Equation2 and also the system's dynamic model.

Briefly, each manifold, which reduces the system order, submits a new constraint. Suitable choice of the macro-variables and their characteristics enables the designer to achieve the targeted performances for the system design including stability and parameter sensitivity.

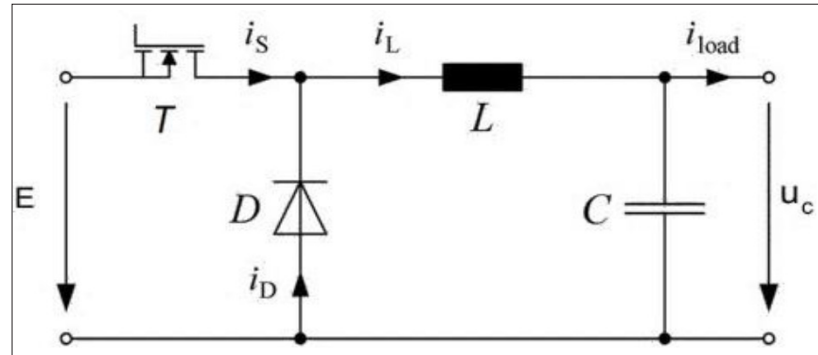

Figure 1. DC Buck converter schematics

Consequently, the synergetic control theory ensures global stability, i.e., once the system reaches the manifold, it stays on it even for large signal fluctuations [14].

\section{DC-DC Buck Converter Modelling}

A primary DC buck converter circuit is shown in Figure 1, consisting of a resistor $R$, a capacitor $C$, a self-inductance $L$, a fast diode, and an IGBT or MOSFET transistor which implements the switching action in the circuit.

When the converter operates in continuous conduction mode (CCM), the system is shown as follows $[19,15]$ :

$\left[\begin{array}{l}i_{L} \\ \dot{u}_{C}\end{array}\right]=\left[\begin{array}{ll}0 & -\frac{1}{L} \\ \frac{1}{C} & -\frac{1}{R C}\end{array}\right]\left[\begin{array}{l}l_{L} \\ u_{C}\end{array}\right]+\left[\begin{array}{c}\frac{E}{L} \\ o\end{array}\right] u$

When the switching state $u$ is equal to one, the switch turns on, and when $u=0, T$ is off.

Equation 4 (below) represents the output voltage $u_{c}$ and its derivative as the system state variables:

$\left\{\begin{array}{l}x_{1}=u_{C} \\ x_{2}=\frac{u_{C}}{d t}\end{array}\right.$

If the ripples are small with the switching frequency being relatively high and the duty ratio of the switching cycle being $d$, then the state space average model can be presented as: [19]:

$\left\{\begin{array}{c}\dot{x}_{1}=x_{2} \\ \dot{x}_{2}=-\frac{x_{1}}{L C}-\frac{x_{2}}{R C}+\frac{E}{L C} d\end{array}\right.$

Taking into account the disturbances generated by parametric variation, the dynamic model of the buck converter can be adjusted as:

$\left\{\begin{array}{c}\dot{x}_{1}=x_{2} \\ \dot{x}_{2}=-\frac{x_{1}}{L C}-\frac{x_{2}}{R C}+\frac{E}{L C} d+F\end{array}\right.$ 
where $\mathrm{F}$ represents all the disturbances which the system can endure.

\section{Non-Singular Terminal Synergetic Control}

Initially, we considered the DC Buck converter modeled in Equation 5 to design a non-singular terminal SYC. At that point, we did not consider the disturbance effect. If we suppose the reference tracking voltage to be, then the tracking error and its derivative are as follows:

$e=x_{1}-y_{\text {ref }}$

$\dot{e}=x_{2}-\dot{y}_{\text {ref }}$

The non-linear macro-variable function of this Non-singular Terminal Synergetic Control is chosen as:

$\Psi(e)=\frac{p}{q} e+\frac{1}{B} \dot{e}^{\frac{p}{q}}=\frac{p}{q}\left(x_{1}-y_{r e f}\right)+\frac{1}{B}\left(x_{2}-\dot{y}_{r e f}\right)^{\frac{p}{q}}$

where $\beta>0, p$ and $q$ are positive odd constants, and Equation 10 must be satisfied.

$1<\frac{p}{q}<2$

When the system reaches the macro-variable, $\psi=0$, its dynamics can be by:

$\Psi=0 \Rightarrow \dot{e}=-\left(B \frac{p}{q}\right)^{\frac{q}{p}} e^{\frac{q}{p}}$

Note that Equation 11 reduces to $\dot{e}=-B$ e, for $p / g=1$, which is the form of conventional synergetic control as in [14].

It was shown in [20], that $\mathrm{e}=0$ is a terminal attractor for Equation (11), which we can rewrite as;

$d t=-\frac{d e}{\left(B \frac{p}{q}\right)^{\frac{q}{p}} e^{\frac{q}{p}}}$

Taking integral of both sides of Equation 12 on the time interval $\left(\mathrm{e}(0) \neq 0, \mathrm{e}\left(t_{s}\right)=0\right)$ gives the following equation [6]:

$t_{S}^{T S Y C}=\frac{p}{\left(B \frac{p}{q}\right)^{\frac{q}{p}}(p-q)}|e(0)|^{\left(1-\frac{q}{p}\right)}$

Equation (13) indicates that, when the system reaches the terminal synergetic mode at $t=t_{r}$, the system state error converges to zero in finite-time $t_{S}^{T S Y C}$.

In Equation 5, $\mathrm{f}=-\frac{x_{1}}{L C}-\frac{x_{2}}{R C^{\prime}}$ and $g=\frac{E}{L C} d$ are known, and the control law is easily derived: $d=\frac{1}{g(x)}\left[-f(x)+\ddot{y}_{r e f}+\ddot{e}\right]$

Using the synergetic approach,

$T \dot{\Psi}+\frac{1}{B} \frac{p}{q} \dot{e}^{\left(\frac{p}{q}-1\right)} \Psi=0$

where

$0<\frac{p}{q}-1<1$

By substituting (7), (8) in (15), and because $\beta>0, p$ and $q$ are positive odd constants, when $x_{2}-\dot{y}_{\text {ref }} \neq 0$ there exists [19,21]:

$\left(x_{2}-\dot{y}_{r e f}\right)^{\left(\frac{p}{q}-1\right)}>0$

Thus,

$\frac{1}{\mathrm{~B}} \frac{p}{q} \dot{e}^{\left(\frac{p}{q}-1\right)}>0$

It is therefore easy to derive the terminal synergetic control law given by:

$d=\frac{1}{g(x)}\left[-f(x)-\frac{1}{T} \Psi(e)-B \dot{e}^{\left(2-\frac{p}{q}\right)}+\ddot{y}_{r e f}\right]$

To prove stability, we chose the following Lyapunov function candidate:

$V=\frac{1}{2} \Psi(e)^{2}$

After differentiation, this leads to:

$\dot{V}=\Psi(e) \dot{\Psi}(e)=-\frac{1}{T} \frac{1}{\mathrm{~B}} \frac{p}{q} \dot{e}^{\left(\frac{p}{q}-1\right)}$

Because Equation 18 is satisfied and $\mathrm{T}>0$, stability is therefore guaranteed for we now have:

$\dot{V} \leq 0$

Generally, the nonlinear system functions $f$ and $g$ are difficult to determine precisely, thus parameter variations and uncertainties can be lumped as $\mathrm{F}$ as in Equation 6.

\section{Adaptive Nonsingular Terminal Synergetic Design}

Here we desire to strengthen system control robustness against the lumped disturbance $F$, which is estimated in an adaptive scheme.

Let the estimated error be defined as:

$\tilde{F}=F-\hat{F}$ 
Where the estimation of $\mathrm{F}$ is $\widehat{\mathrm{F}}$ and let a Lyapunov candidate function be defined as:

$V=\frac{1}{2} \Psi^{2}+\frac{1}{2 \gamma} \tilde{F}^{2}$

Taking the derivative of Lyapunov function candidate $V$, gives the following equation:

$$
\begin{array}{r}
\dot{V}=\Psi(e)\left[\left(\frac{p}{q} \dot{e}+\frac{1}{B} \frac{p}{q} \dot{e}^{\left(\frac{p}{q}-1\right)}(f(x)+g(x) d+\hat{F}-\right.\right. \\
\left.\left.\left.\ddot{y}_{r e f}\right)\right)-\frac{1}{\gamma} \hat{F}\left(\dot{\hat{F}}-\gamma \Psi \frac{1}{B} \frac{p}{q} \dot{e}^{\left(\frac{p}{q}-1\right)}\right)\right]
\end{array}
$$

The influence of the estimation error is eliminated by choosing the following adaptive law.

$\dot{\hat{F}}=\gamma \Psi \frac{1}{B} \frac{p}{q} \dot{e}^{\left(\frac{p}{q}-1\right)}$

The control input is therefore given as

$$
\begin{gathered}
d=\frac{1}{g(x)}\left[-\frac{1}{T} \Psi(e)-f(x)-B \dot{e}^{\left(2-\frac{p}{q}\right)}+\ddot{y}_{r e f}-\hat{F}-\right. \\
\left.-w \Psi^{\frac{m}{n}}-h \Psi\right]
\end{gathered}
$$

Where $w>0, h>0, m, n$ are positive odd constants and $m<n$.

In Equation $(19,27)$, it is evident that as long as $1<p / q<2$, the main drawback of ANTSYC has been avoided [19].

Substituting (27) and (26) into (25) leads to;

$\dot{V}=\frac{1}{B} \frac{p}{q} \dot{e}^{\left(\frac{p}{q}-1\right)}\left[-\frac{1}{T} \Psi^{2}-w \Psi^{\left(\frac{m+n}{n}\right)}-h \Psi^{2}\right]$

Because $m<n$ and $m, n$ are positive odd constants, Equation (16) is satisfied, so when $\psi \neq 0$, the following condition is satisfied [19].

$\Psi\left(\frac{m+n}{n}\right)>0$

Therefore, with the condition of $\dot{\mathrm{e}} \neq 0$, the following inequality is satisfied;

$\dot{V}=\frac{1}{B} \frac{p}{q} \dot{e}^{\left(\frac{p}{q}-1\right)}\left[-\frac{1}{T} \Psi^{2}-w \Psi^{\left(\frac{m+n}{n}\right)}-h \Psi^{2}\right] \leq 0$.

So Lyapunov stability can be satisfied.

Substituting control input (27) into model (6) leads to:

$\dot{x}_{2}-\ddot{y}_{r e f}=-\frac{1}{T} \Psi-B \dot{e}^{\left(2-\frac{p}{q}\right)}-w \psi^{\frac{m}{n}}-h \Psi+F-\hat{F}$

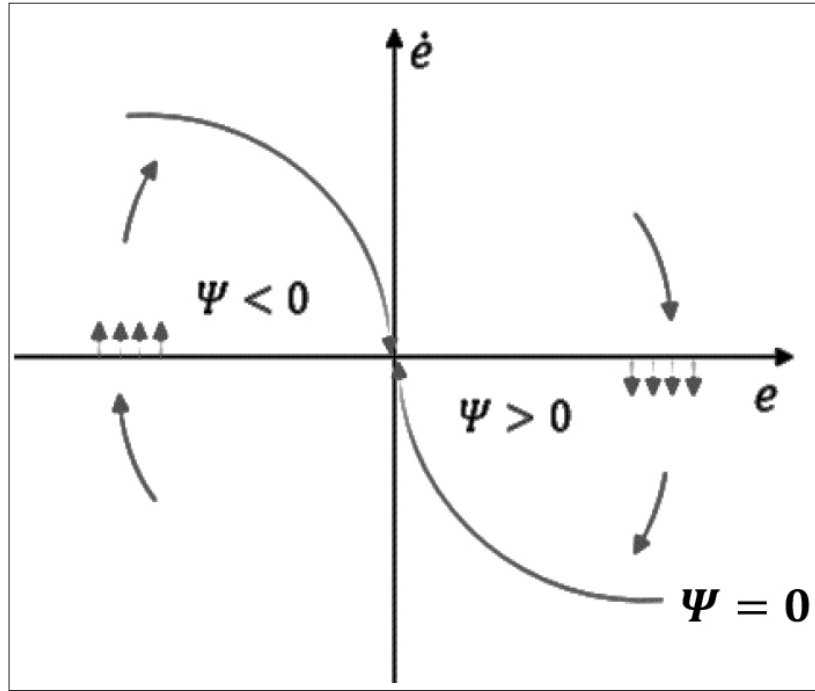

Figure 2. System phase portrait

By transforming it, it can be rewritten as:

$\dot{x}_{2}-\ddot{y}_{r e f}=F-\hat{F}-\frac{1}{T} \Psi(e)-w \Psi^{\frac{m}{n}}-h \Psi$

Suppose that $x_{2}-\dot{y}_{\text {ref }}=0$ is satisfied, it can be seen from Figure 2 that if $\Psi>0$, the control system can reach synergetic macro-variable only when $\dot{\mathrm{x}}_{2}-\ddot{y}_{\text {ref }}<0$ comes into existence. This requires the condition of $F-\hat{F}<\frac{1}{T} . \Psi(e)++w \Psi^{\frac{m}{n}}+h \Psi$ is satisfied when $\Psi>0$. Similarly, $F-\hat{F}>\frac{1}{T} \Psi(e)++w \Psi^{\frac{m}{n}}+h \Psi$ is satisfied when $\Psi<0$. By adjusting parameters of the control system, the above-mentioned conditions can be satisfied.

Figure 3 shows the simulated responses of inductor current (iL), output voltage Vo, error, and derivative error, obtained by the NTSYC method for $R=10 \Omega$ using $B=400$, and $p / q=1.66$. It can be seen from Figure 3 that singularity is avoided by the NTSC method, which overcomes the oscillations on the output responses. The main reason for this avoidance comes from the fact that there is no singular term in the control law expression Equaiton 27.

\section{Simulation Results}

In order to evaluate, the behavior of the control obtained (ANTSY) was examined with a DC-DC buck converter by simulation.

the parameters of the controller are; $T=1 e-6, B=400$,

$p / q=1.66, m / n=0.025, w=2 e 3, h=1 e 3, \gamma=50$.

Simulations are carried out using the Simulink of Matlab/Simulink (2014a) shown in the picture Figure 4 with a step size of 0.2 us. 

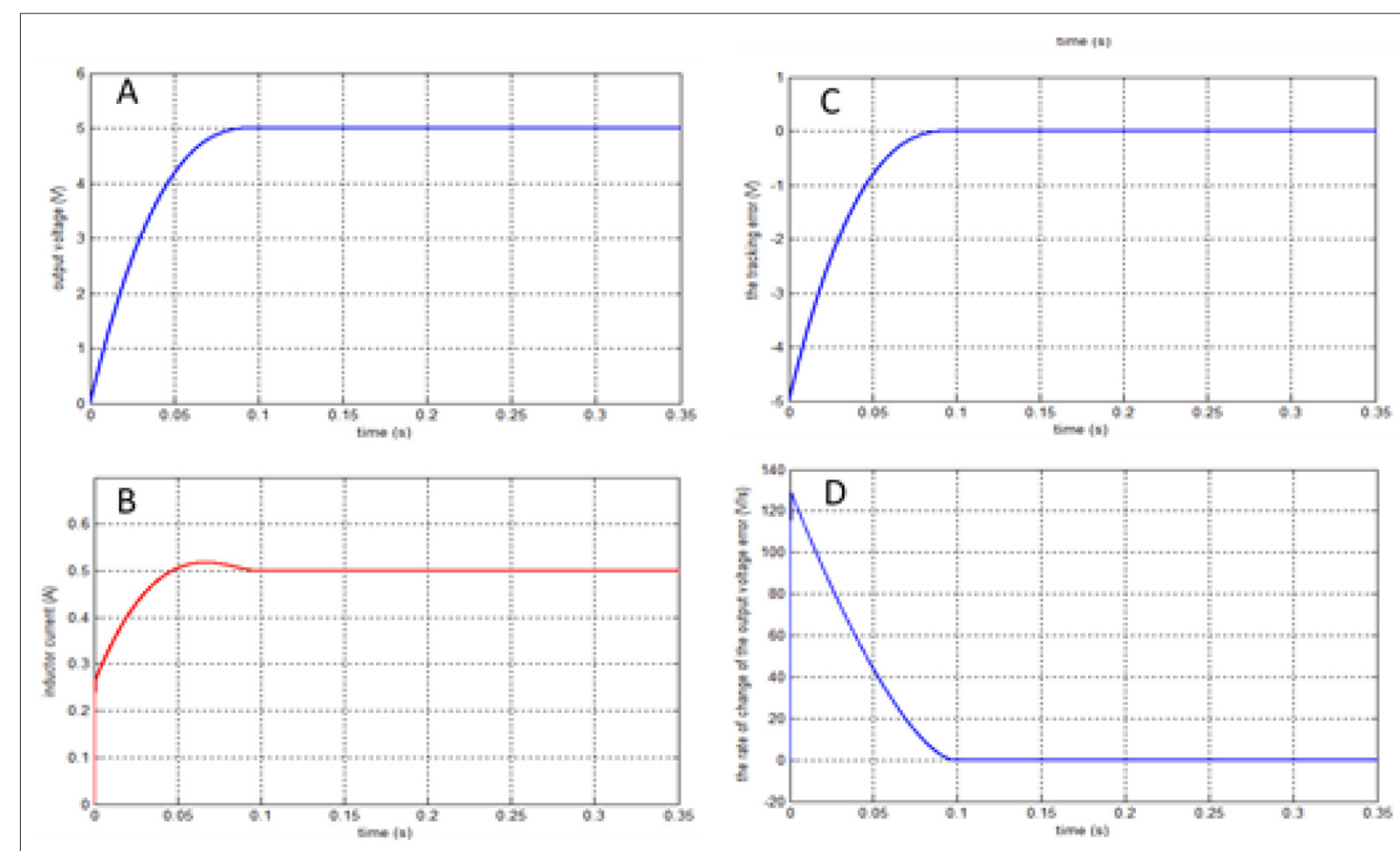

Figure 3. a-d. Response of, output voltage (uc) (A); inductor current (iL) (B); the output voltage error (err) (C); the rate of change of the output voltage error (derr) (D), obtained by ATSYC method

Table 1. Parameters of the DC buck converter.

\begin{tabular}{ll}
\hline Description & Nominal value \\
\hline Inductance (L) & $13 \mathrm{mH}$ \\
\hline Capacitance (C) & $2000 \mathrm{uF}$ \\
\hline Load resistance (R) & $10-5 \Omega$ \\
\hline Reference output voltage(yref) & $5-7 \mathrm{~V}$ \\
\hline Input voltage (E) & $10-9 \mathrm{~V}$ \\
\hline
\end{tabular}

The parameters of the buck converter are given in Table 1.

Figure 5 presents the simulated results when the variation takes place in input voltage $\mathrm{E}$, at $\mathrm{t}=0.2 \mathrm{~s}$ from $10 \mathrm{~V}$ to $9 \mathrm{~V}$ in The proposed controller gives excellent performances during the step changes in R from $10 \Omega$ to $5 \Omega$ in Figure 5(a), and $y_{\text {ref }}$ from 5 $\mathrm{V}$ to $7 \mathrm{~V}$ Figure $5(\mathrm{~b})$. In addition, Figure 5 (c, d), obtained by the NTSYC and NTSMC, these two methods result in a small steadystate error. However, the NTSC method leads to less steadystate error than the NTSC method.

The trajectory in Figure 6 (a), was obtained with $B=400$ and different values of $(p / q)$. It is clear that when $(p / q)$ is high, the inclination of the macro-variable line is small, and consequently there is a slower dynamic response. Furthermore, we get faster responses whenever the value of $(p / q)$ is small.

In Figure $6(b)$ the trajectory is obtained with $(p / q=1.66)$ and different values of $\mathrm{B}$. Clearly, there is an Inverse relationship between (B) and the slope of $\psi$ line, but an overshoot probably occurs when the value of $B$ is too high, and even with the high value of $B$, still no shattering problem on the output voltage although, fast convergence.

Figure 7 shows the simulated responses of the output voltage and the inductor current iL to step load resistance changes in R from $10 \Omega$ to $5 \Omega$, from $5 \Omega$ to $10 \Omega$, and from $10 \Omega$ to $5 \Omega$, respectively. At $t=0.1 \mathrm{~s}$, the first step load change occurs, and as a result, the inductor current iL changes from $1 \mathrm{~A}$ to $0.5 \mathrm{~A}$ and an acceptable overshoot appears in the output voltage responses. The second load variation occurs at $t=0.2 \mathrm{~s}$ which causes a change in iL from $0.5 \mathrm{~A}$ to $1 \mathrm{~A}$ and a drop in the output voltage. And the same behavior mentioned in the first load change appears in the third load change. These results clearly show the robust performance of the NTSMC method.

\section{Conclusion}

First, the Terminal Synergetic approach shows that we can achieve proper performance with regard to response time and 

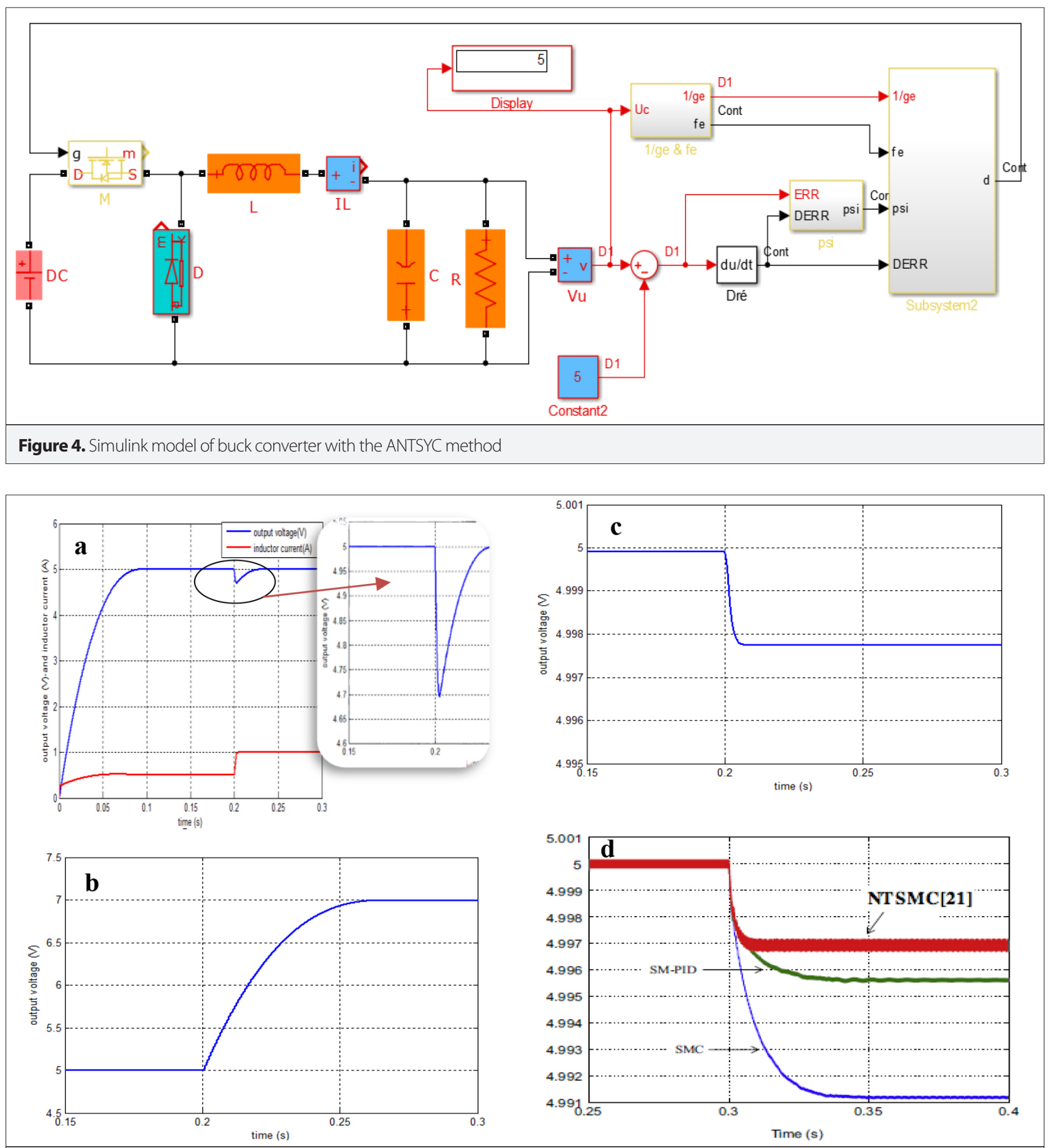

Figure 5. a-d. Output voltage responses due to the step changes in R, yref and E, obtained via NTSYC methods: Step change in R from $10 \Omega$ to 5 $\Omega$ (a); Step change in yref from $5 \mathrm{~V}$ to $7 \mathrm{~V}(\mathrm{~b})$ and Step change in E from $10 \mathrm{~V}$ to $9 \mathrm{~V}$ with ANTSYC (c); Step change in E from 10V to 9V with ANTSMC (d)

overshoot, but during this study we discovered the main disadvantage of the synergetic theory, namely, it uses a model of the system for control synthesis, which was solved by an adaptive estimation method.

As a result, we have proposed a novel "no singular terminal adaptive synergetic" DC-DC buck converter controller and eval- uated its performance thoroughly using simulation for different perturbed operating conditions.

Our results indicate that with this design one can attain proper tracking, and insensitivity to perturbations. Concerning robustness, the results obtained in the existence of load and input voltage changes also show excellent performance. 


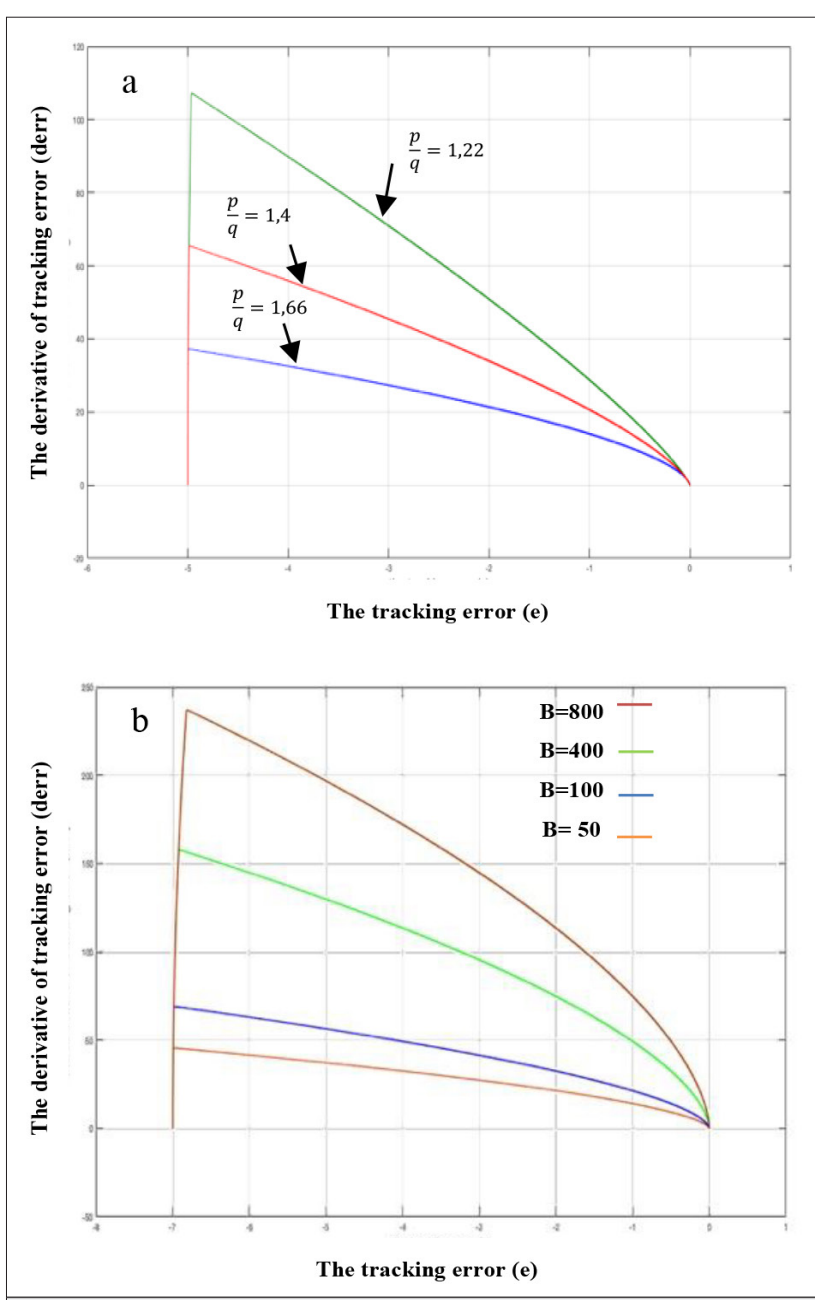

Figure 6. a, b. State trajectories with different values of $(p / q)$ when $B$ is constant (a); State trajectories with different values of $B$ when $(p / q)$ is constant (b)

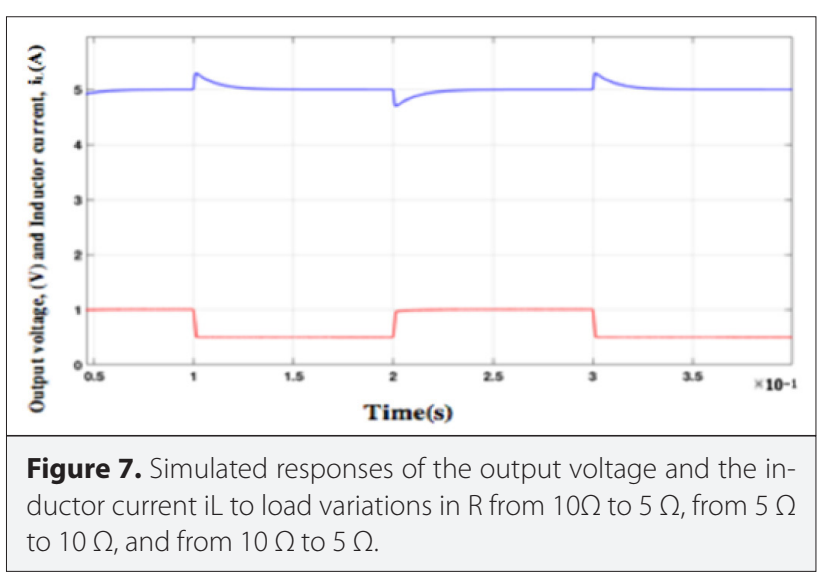

We consider that our suggested robust approach could be implemented easily, as it does not rely on a discontinuous control component in contrast to SMC. Therefore, we aim to experimentally validate the controller developed in this work in a future study.
Peer-review: Externally peer-reviewed.

Conflict of Interest: The authors have no conflicts of interest to declare.

Financial Disclosure: The authors declared that this study has received no financial support.

\section{References}

1. J. E. Slotine, W.P. Li, "Applied nonlinear control", Prentice-Hall, Englewood Cliffs, NJ, 1991.

2. A. Levant, "Higher-order sliding modes, differentiation, and output-feedback control," Int J Control, vol. 76, no 9-10, 924-941, 2003.

3. V. I. Utkin, "Sliding modes in control and optimization", New York, Springer, 1992.

4. H. F. Ho, Y. K. Wong, A. B. Rad, "Adaptive fuzzy sliding mode control with chattering elimination for nonlinear SISO systems", Simulation Modelling Practice and Theory, vol. 17, no. 7, pp. 1199-1210, 2009.

5. Y.D. Son, T.W. Heo, E. Santi, A. Monti, “Synergetic control approach for induction motor speed control", $30^{\text {th }}$ Annual Conference of the IEEE Industrial Electronics Society, 2004.

6. M. Zhihong, A.P. Paplinski, H.R. Wu, "A robust MIMO terminal sliding mode control scheme for rigid robotic manipulator", IEEE Transactions on Automatic Control, vol. 39, no. 12, pp. 2464-2469, 1994.

7. F. Liping, Y. Yazhou, K Boshnakov, "Adaptive backstepping based terminal sliding mode control for DC-DC convertor", International Conference on Computer Application and System Modelling (ICCASM 2010), 2010, pp. 323-327.

8. D. Zhao, S. Li, F. Gao, "A New Terminal Sliding Mode Control for Robotic Manipulators", Int J Control, vol. 82, no. 10, pp.1804-1813, 2009.

9. M. Jin, J. Lee, P. H. Chang, C. Choi, “Practical Nonsingular Terminal Sliding-Mode Control of Robot Manipulators for High-Accuracy Tracking Control", IEEE Transactions on Industrial Electronics, vol. 56, no. 9, pp. 3593-3601, 2009.

10. Y. Feng, S. Bao, X. Yu, "Inverse Dynamics Nonsingular Terminal Sliding Mode Control of Two-Link Flexible Manipulators", International Journal of Robotics and Automation, vol. 19, pp. 91-102, 2004.

11. J. F. Tsai, Y. P. Chen, "Sliding Mode Control and Stability Analysis of Buck DC-DC Converter", Int JElect, vol. 94, no. 3, pp. 209-222, 2007.

12. O. Barambones, P. Alkorta, A. J. Garrido, I. Garrido, F. J. Maseda, "An Adaptive Sliding Mode Control Scheme for Induction Motor Drives", International Journal of Circuits, Systems and Signal Processing, vol. 1, no. 1, pp. 73-79, 2007.

13. F. Qiao, Q. Zhu, A. F. T. Winfield, C. Melhuish, "Adaptive Sliding Mode Control for MIMO Nonlinear Systems based on a Fuzzy Logic Scheme", International Journal of Automation and Computing. vol.1, no. 1, pp. 51-62. 2004.

14. E. Santi, A. Monti, D. Li, K. Proddutur, R. Dougal, "Synergetic control for DC-DC boost converter: implementation options", IEEE Transactions on Industry Applications, vol. 39, no. 6, pp. 1803-1813, 2003.

15. A. A. Kolesnikov, "Modern Applied Control Theory: Synergetic approach in control theory", vol. 2, Russian, Moscow-Taganrog, TSURE Press, 2000.

16. Q. Wang, J. Feng, T. Li, "Analysis of the Synergetic Control Based on Variable Structure and Application of Power Electronics", International Conference on Information Engineering and Computer Science, 2009, pp. 1-4. 
17. E. Santi, A. Monti, D. Li, K. Proddutur, R. A. Dougal, "Synergetic control for power electronics applications: A comparison with the sliding mode approach", J Circuits Syst Comput, vol. 13, no. 4, pp. 737-760, 2004.

18. L. Medjbeur, M. N. Harmas, "Adaptive fuzzy terminal synergetic control", International Conference on Communications, Computing and Control Applications (CCCA), 2011.
19. L. Fan, Y. Yu, "Adaptive Non-singular Terminal Sliding Mode Control for DC-DC Converters", Advances in Electrical and Computer Engineering, vol. 11, no. 2, pp. 119-122, 2011.

20. M. Zak, "Terminal Attractors for Addressable Memory in Neural Networks", Phys Lett A, vol. 133, no. 1-2, pp. 18-22, 1988.

21. H. Komurcugil, "Non-singular terminal sliding-mode control of DC-DC buck converters", Control Engineering Practice, vol. 21, no. 3, pp. 321-332, 2013

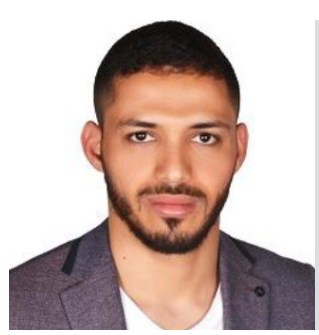

Yakoub Nettari was born in Touggourt, Algeria in 1987. He has obtained his Engineer degree in Electromechanical Engineering from M'sila University in 2010. He has obtained M.Sc degree in Control and Automatic Engineering from Setif University in 2014. He is PhD student in Electrical Engineering at Yıldız Technical University since 2015. His current research is on Robust and intelligent Control, Automation, Robotics, DC-DC converters and Renewable Energy.

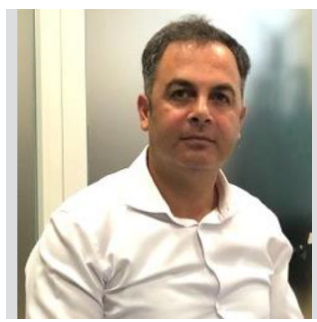

Serkan Kurt was born in Kırıkkale in 1976. He has obtained his B.Sc in Electrical and Electronics Engineering from İstanbul University in 1999. He has obtained M.Sc degree in Computer Engineering from Gebze Technical University in 2002. He has obtained Phd degree in Electrical Engineering in Yıldız Technical University in 2007. His areas of research includes Sensor Network, Control and Automation, Robotics, System Design. He has been working at Yıldız Technical University since 2002. 